\title{
Testing the magnetism of polymerized fullerene
}

\author{
D. W. Boukhvalov, ${ }^{1,2}$ P. F. Karimov, ${ }^{1}$ E. Z. Kurmaev, ${ }^{1}$ T. Hamilton, ${ }^{3}$ A. Moewes,${ }^{3}$ L. D. Finkelstein, ${ }^{1}$ M. I. Katsnelson,,${ }^{1,4}$ \\ V. A. Davydov, ${ }^{5}$ A. V. Rakhmanina, ${ }^{5}$ T. L. Makarova, ${ }^{6}$ Y. Kopelevich, ${ }^{7}$ S. Chiuzbăian, ${ }^{8}$ and M. Neumann ${ }^{8}$ \\ ${ }^{1}$ Institute of Metal Physics, Russian Academy of Sciences-Ural Division, 620219 Yekaterinburg GSP-170, Russia \\ ${ }^{2}$ Forschungszentrum Jülich, D-52425 Jülich, Germany \\ ${ }^{3}$ Department of Physics and Engineering Physics, University of Saskatchewan, 116 Science Place, \\ Saskatoon, Saskatchewan, Canada S7N 5E2 \\ ${ }^{4}$ Uppsala University, Department of Physics, Box 530, SE-751 21 Uppsala, Sweden \\ ${ }^{5}$ Institute of High Pressure Physics, 14209 Troitsk, Russia \\ ${ }^{6}$ Department of Experimental Physics, Umea University, S-90187, Sweden \\ ${ }^{7}$ Instituto de Fisica, Unicamp, 13083-970 Campinas, Sao Paulo, Brazil \\ ${ }^{8}$ Universität Osnabrück, Fachbereich Physik, D-49069 Osnabrück, Germany
}

(Received 16 August 2003; published 24 March 2004)

\begin{abstract}
We present band structure calculations of rhombohedral $\mathrm{C}_{60}$ performed in the local-spin-density approximation. Rhombohedral $\mathrm{C}_{60}\left(\mathrm{Rh}-\mathrm{C}_{60}\right)$ is a two-dimensional polymer of $\mathrm{C}_{60}$ with trigonal topology. No magnetic solution exists for $\mathrm{Rh}_{-} \mathrm{C}_{60}$ and energy bands with different spins are found to be identical and not split. The calculated C $2 p$ partial density of states is compared to carbon $K$-edge x-ray emission and absorption spectra and shows good agreement. It is concluded that the rhombohedral distortion of $\mathrm{C}_{60}$ itself cannot induce magnetic ordering in the molecular carbon. The result of magnetization measurements performed on the same Rh- $\mathrm{C}_{60}$ sample corroborates this conclusion.
\end{abstract}

DOI: 10.1103/PhysRevB.69.115425

PACS number(s): 78.70.En, 61.48.+c, 71.20.Tx

A recent report on ferromagnetic ordering in samples synthesized at high pressures and temperatures, on the basis of the rhombohedral polymerized phase, ${ }^{1}$ has stimulated interest in carbon systems as possible new magnetic materials made exclusively of light elements. Reports on magnetism in organic compounds and systems composed of nonmagnetic constituent elements are naturally met with skepticism due to the possible influence of magnetic impurities, ${ }^{2-4}$ whereas an intrinsic origin of the ferromagnetism in both graphite and rhombohedral $(\mathrm{Rh})-\mathrm{C}_{60}$ has been demonstrated.

In order to investigate the possibility of long-range magnetic ordering in the rhombohedral $\mathrm{C}_{60}$ polymer we have performed electronic structure calculations that employ density functional theory in the local-spin-density approximation (LSDA). ${ }^{5,6}$ The results, consistent with x-ray fluorescence measurements, do not show a magnetic solution and we conclude that a simple distortion of $\mathrm{C}_{60}$ molecules in the $\mathrm{Rh}-\mathrm{C}_{60}$ polymer cannot induce magnetic ordering in the system.

Spin-polarized band structure calculations have been performed for rhombohedral $\mathrm{C}_{60}$ molecules using a selfconsistent linearized muffin-tin orbital (LMTO) method within the local density approximation (computer code TBLMTO-47, see Ref. 7). This method has been successfully used for different $\mathrm{C}_{60}$ compounds. ${ }^{8,9}$ For comparison, nonspin-polarized band structure calculations of $\mathrm{C}_{60}$ have been carried out. We tried, as a first iteration, different sets of initial spin polarizations of $2 p$ carbon states corresponding to different hypothetical magnetic structures, but this polarization inevitably tends to zero at the iterations of selfconsistency procedure and the final state turns out to be nonmagnetic (Fig. 2). The isolated $\mathrm{C}_{60}$ molecule has an icosahedral symmetry and it is usually assumed that the same holds for each molecule in the solid, monomeric fcc and simple cubic phases. ${ }^{8}$ The positions of atoms in cubic $\mathrm{C}_{60}$ are taken from Ref. 8. Rh- $\mathrm{C}_{60}$ clusters form a trigonal lattice in each layer, and the space group of this system is $R \overline{3} \mathrm{~m}$. The crystal lattice structure in this case is usually expressed by using hexagonal system parameters: $a=9.19$ and $c$ $=24.5 \AA$; the interlayer distance is $c / 3 .^{10}$ The electronic structure calculations have been carried out in a standard way, but two important points should be mentioned. First, this is a problem of an optimal choice of atomic radii, numbers of empty spheres, and overlap parameters. We have used the method described in Ref. 9 to solve this problem. Second, a large sized elementary cell in real space permits us to use fewer $k$ points. It appeared that $13 k$ points in the irreducible part of the Brillouin zone are sufficient for convergence of the density of states picture. One can see in Fig. 3 that the latter is in a good agreement with the experimental data.

Soft x-ray emission measurements of cubic and rhombohedral $\mathrm{C}_{60}$ were carried out at Beamline 8.0.1 of the Advanced Light Source at Lawrence Berkeley National Laboratory. For the emission spectra, the emitted radiation is collected in a Rowland-circle type spectrometer with spherical gratings and recorded with an area-sensitive multichannel detector. ${ }^{11}$ This combination provides an instrumental resolution of about $0.4 \mathrm{eV}$ at $\mathrm{C} K \alpha$ emission energies.

The x-ray photoemission spectroscopy (XPS) measurements were carried out with a PHI 5600 ci multi-technique spectrometer using monochromatized $\mathrm{Al} K \alpha$ radiation $\left(E_{\mathrm{exc}}\right.$ $=1486.6 \mathrm{eV}$ ). The estimated energy resolution is $0.35 \mathrm{eV}$ and the base pressure in the vacuum chamber during measurements was about $5 \times 10^{-9}$ Torr.

The measured $\mathrm{Rh}-\mathrm{C}_{60}$ sample has a cylinder geometry, mass $m=16 \mathrm{mg}$, and was synthesized at the limit of thermal stability of the rhombohedral polymerized $\mathrm{C}_{60}$ phase at $T$ $=800^{\circ} \mathrm{C}$ and pressure $\mathrm{P}=6 \mathrm{GPa}$ from the powder fullerite $\mathrm{C}_{60}$ using a toroid-type high-pressure apparatus. Complementary magnetization $\vec{M}(\vec{H}, T)$ measurements were performed using the SQUID magnetometer MPMS5 (Quantum 


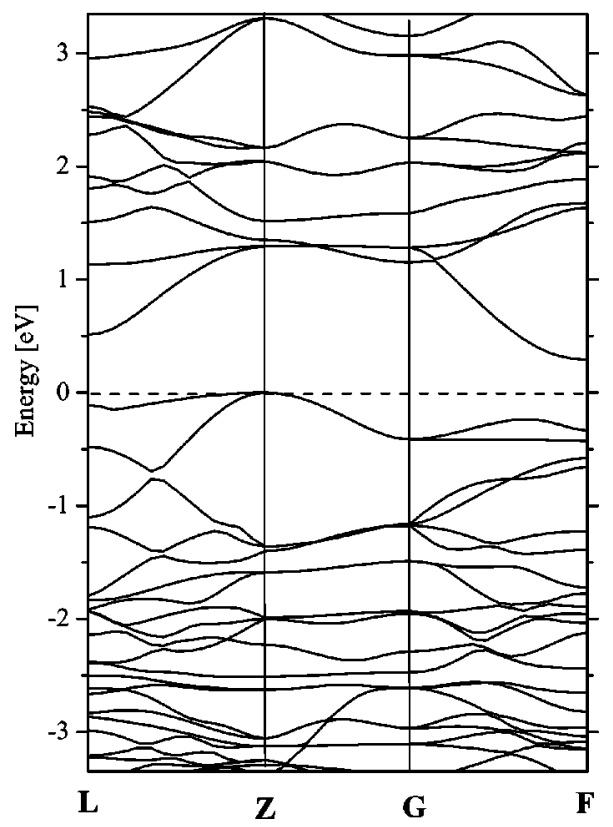

FIG. 1. Energy bands for rhombohedral $\mathrm{C}_{60}$.

\section{Design).}

The calculated electronic band structure for the rhombohedral polymer of $\mathrm{C}_{60}$ is shown in Figs. 1 and 2 (the Fermi level is set to zero in both figures). The energy gap between the top of the valence band and the bottom of the conduction band is $0.29 \mathrm{eV}$, which is in accordance with previous band structure calculations. ${ }^{12}$ No stable ferromagnetic solutions showing energy splitting between spin-up and spin-down electrons were found. Thus, the pure rhombohedral polymerized phase of $\mathrm{C}_{60}$ does not appear to be magnetic according to the LSDA approach.

Generally speaking, the applicability of the LSDA to fullerene-based systems is not obvious because of the possible contribution from correlation effects (for example, see Ref. 13). In order to verify the accuracy of our conclusions we compared the calculated C $2 p$ density of occupied states to carbon $K \alpha$ XES of cubic and rhombohedral $\mathrm{C}_{60}$ as shown

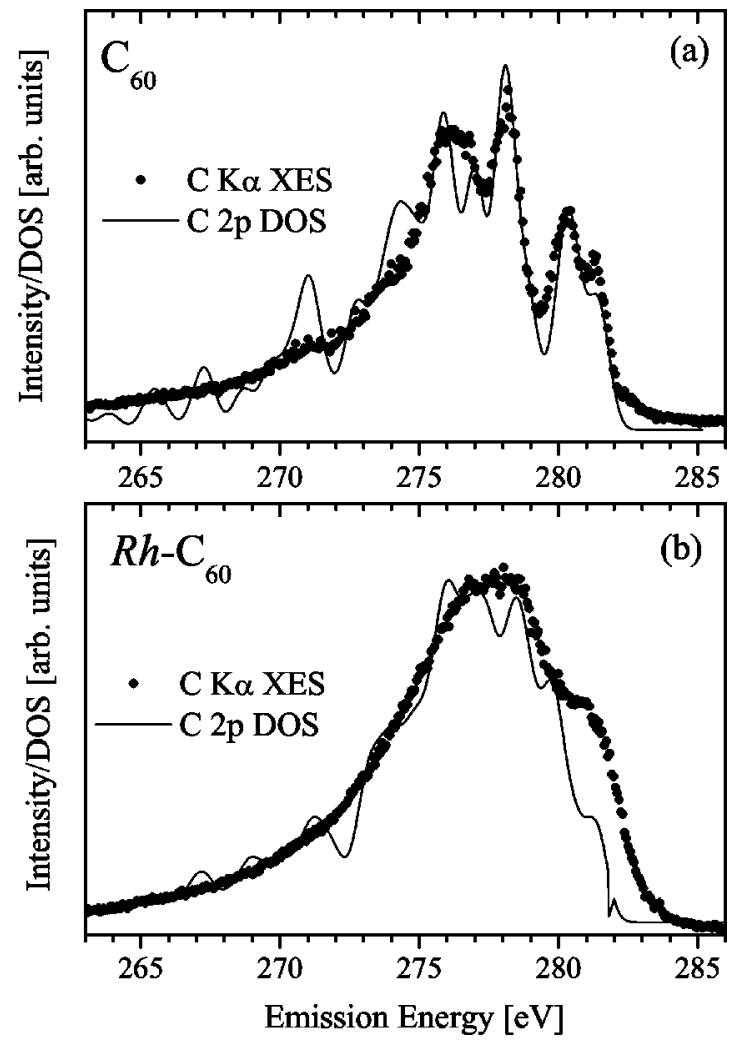

FIG. 3. Comparison of C $2 p$ density of states (DOS) and C $K \alpha$ XES of (a) cubic and (b) rhombohedral $\mathrm{C}_{60}$. Calculated C $2 p$ DOSs are smeared by Gaussian curves with full widths at half maximum of $0.35 \mathrm{eV}$.

in Fig. 3. Clearly, experimental carbon $K$-emission spectra are reproduced quite well by theoretical $\mathrm{C} 2 p$ density of states calculations for both structural modifications of $\mathrm{C}_{60}$. The broader features present in the measured spectra are due to life-time broadening and not an instrumental effect. As displayed in Fig. 4 there are more sharp features in the cubic $\mathrm{C}_{60}$ XPS valence-band (VB) spectrum than that of the rhombohedral phase. This is due to the higher space symmetry in cubic $\mathrm{C}_{60}$. The sharp features are more characteristic of small

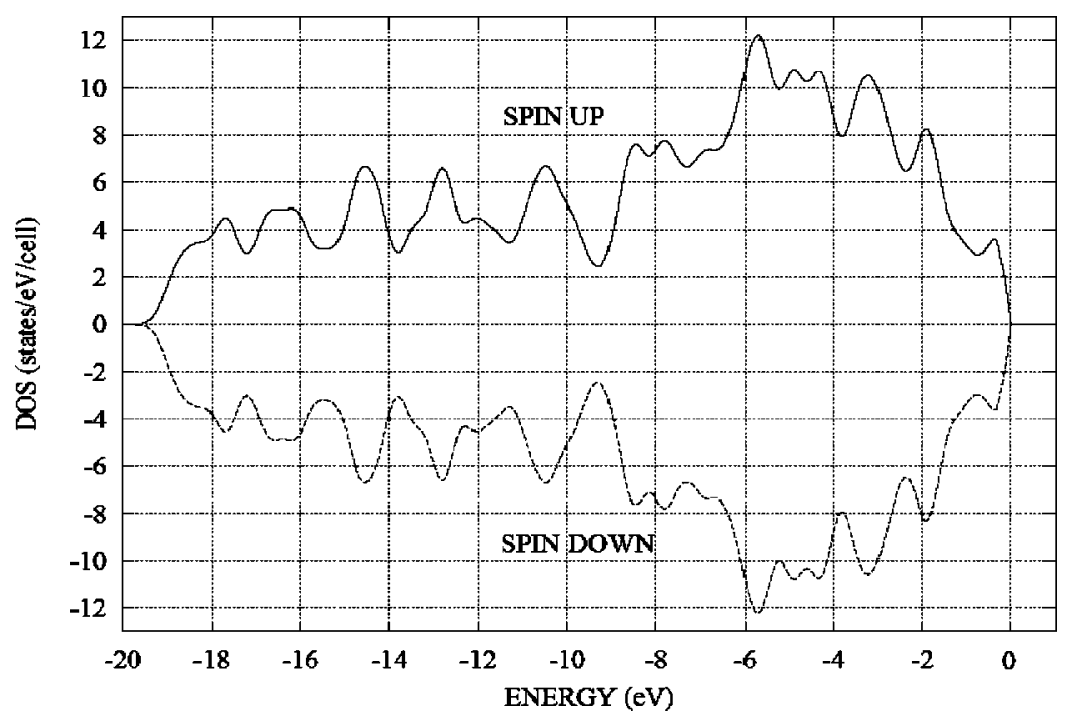

FIG. 2. Density of states for $\mathrm{Rh}-\mathrm{C}_{60}$. 


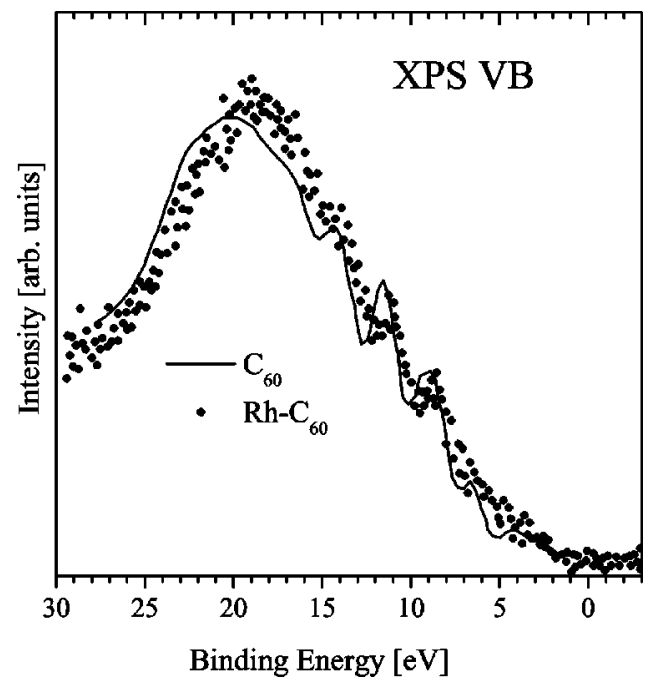

FIG. 4. Comparison of XPS VBs with calculated DOS of cubic and rhombohedral $\mathrm{C}_{60}$. Calculated DOS are weighted by atomic $\mathrm{C}$ $2 s$ and $\mathrm{C} 2 p$ photoionization cross sections. The XPS VB of cubic $\mathrm{C}_{60}$ is taken from Ref. 15.

molecules than of solids, showing that the weak intermolecular interactions have only a slight effect on the density of states. The main effect of the interactions between $\mathrm{C}_{60}$ molecules in the polymer is a broadening of subbands located at the top of the valence band, which is seen in carbon $K$-emission spectra. According to the orbital analysis, the subbands at the top of the valence band largely stem from $\pi$ states and the deeper subbands are almost entirely $\sigma$ derived. Both experimental C $K \alpha$ XES and XPS VB spectra are very close to the calculated energy bands of occupied electronic states, which demonstrates reasonable agreement between theory and experiment. Thus, LSDA band structure calculations are adequate for this case and our conclusion about the absence of ferromagnetic solutions is reliable. This conclusion agrees with the results of magnetization measurements ${ }^{14,15}$ as well as theoretical calculations, ${ }^{16}$ which indicate that the ferromagnetic behavior of fullerenes and related materials may originate from various defects. ${ }^{14-16}$

The magnetization measurements performed on the $\mathrm{Rh}-\mathrm{C}_{60}$ sample studied in this work provide additional information about the inhomogeneous character of the ferromagnetism. Figure 5 presents $M(T)$ measured at $H=100 \mathrm{Oe}$ in both zero-field-cooled (ZFC) and field-cooled (FC) cooling regimes. The magnetization data corresponding to the $\mathrm{ZFC}$ regime, $M_{Z F C}(T, H)$, were taken on heating after the sample cooling at $H=0$, and the magnetization in the $\mathrm{FC}$ regime, $M_{F C}(T, H)$, was measured as a function of decreasing temperature in the applied field. The difference between $M_{Z F C}(T, H)$ and $M_{F C}(T, H)$ together with the observation of ferromagnetic-type $M(H)$ hysteresis loops (Fig. 5, inset) suggests that our sample possesses a disordered magnetism originating from isolated ferromagnetic clusters. The results of $\mathrm{X}$-ray structural analysis performed on this sample, shown in Fig. 6, reveal the coexistence of a $\mathrm{Rh}-\mathrm{C}_{60}$ phase and clusters of graphitelike layers, which may be a source of the local ferromagnetism in the nominal $\mathrm{Rh}-\mathrm{C}_{60}$ compound. ${ }^{14}$

To conclude, we have performed $a b$ initio spin polarized

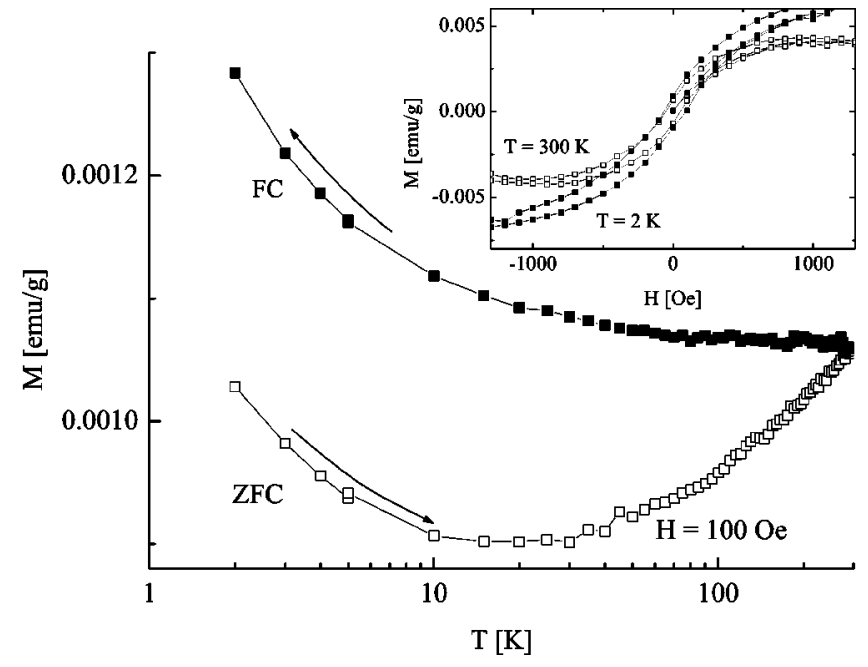

FIG. 5. $M(T, H)$ measured in both ZFC and FC regimes at an applied magnetic field $H=100 \mathrm{Oe}$; the inset demonstrates low-field portions of ferromagnetic-type $M(H)$ hysteresis loops obatined at $T=2$ and $300 \mathrm{~K}$.

band structure calculations and found that $\mathrm{Rh}-\mathrm{C}_{60}$ cannot be ferromagnetic in the LSDA approach. The calculated energy bands are confirmed by x-ray fluorescence measurements, which demonstrate the applicability of the LSDA. Also, the $\mathrm{Rh}-\mathrm{C}_{60}$ phase is not magnetic; the ferromagnetism occurring in the nominal $\mathrm{Rh}-\mathrm{C}_{60}$ samples is associated with other carbon structures that formed when the fullerene cages collapsed. ${ }^{1,14}$

Funding by the Research Council of the President of the Russian Federation (Grant Nos. NSH-1026.2003.2 and NSH747.2003.2), Russian Science Foundation for Basic Research (Project Nos. 02-02-16674 and 03-03-32640), FAPESP, $\mathrm{CNPq}$ and the Natural Sciences and Engineering Research Council of Canada (NSERC) is gratefully acknowledged. The work at the Advanced Light Source at Lawrence Berkeley National Laboratory was supported by U.S. Department of Energy (Contract No. DE-AC03-76SF00098).

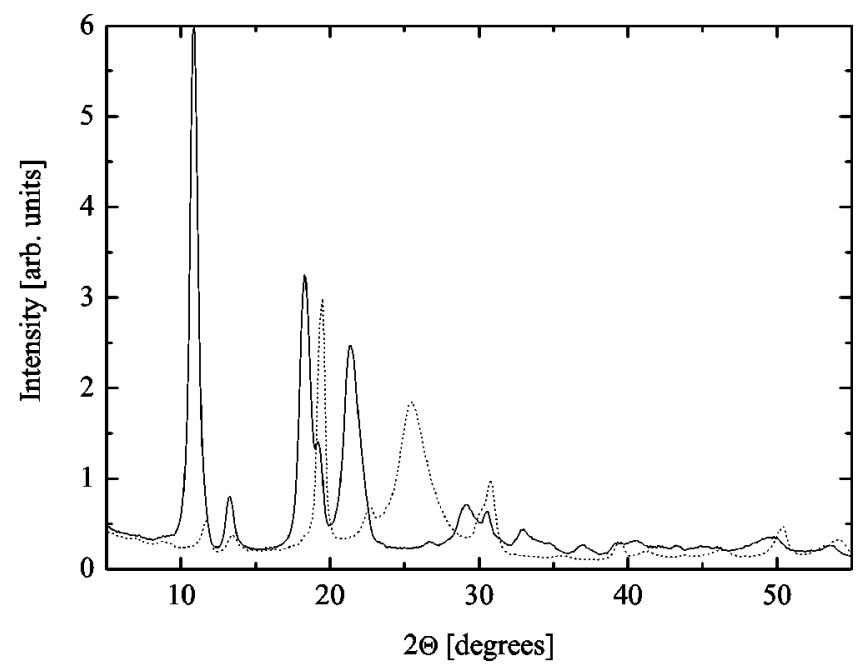

FIG. 6. X-ray diffraction patterns measured for two mutually perpendicular orientations of the sample. 
${ }^{1}$ T.L. Makarova, B. Sundqvist, R. Höhne, P. Esquinaz, Y. Kopelevich, P. Scharff, V.A. Davydov, L.S. Kashevarova, and A.R. Rakhmanina, Nature (London) 413, 716 (2001).

${ }^{2}$ Y. Kopelevich, P. Esquinazi, J.H.S. Torres, and S. Moehlecke, J. Low Temp. Phys. 119, 691 (2000).

${ }^{3}$ P. Esquinazi, A. Setzer, R. Höhne, C. Semmelhack, Y. Kopelevich, D. Spemann, T. Butz, B. Kohlstrunk, and M. Lösche, Phys. Rev. B 66, 024429 (2002).

${ }^{4}$ K.H. Han, D. Spemann, R. Höhne, A. Setzer, T. Makarova, R. Esquinazi, and T. Butz, Carbon 41, 785 (2003).

${ }^{5}$ P. Hohenberg and W. Kohn, Phys. Rev. 136, B864 (1964); W. Kohn and L. Sham, Phys. Rev. 140, A1133 (1964).

${ }^{6}$ U. von Barth and L. Hedin, J. Phys. C 5, 1629 (1972); O. Gunnarsson and B.I. Lundquist, Phys. Rev. B 13, 4274 (1976).

${ }^{7}$ M. Methfessel, M. van Schilfgaarde, and R.A. Casali, in Electronic Structure and Physical Properties of Solids: The Uses of the LMTO Method, edited by H. Dreysse, Lecture Notes in Physics Vol. 535 (Springer-Verlag, Berlin, 2000).

${ }^{8}$ N. Laouni, O.K. Andersen, and O. Gunnarsson, Phys. Rev. B 51,
17446 (1995).

${ }^{9}$ S. Satapathy, V.P. Antropov, O.K. Andersen, O. Jepsen, O. Gunnarsson, and A.I. Liechtenstein, Phys. Rev. B 46, 1773 (1992).

${ }^{10}$ M. Núñez-Regueiro, L. Marques, J.-L. Hodeau, O. Béthoux, and M. Perroux, Phys. Rev. Lett. 74, 278 (1995).

${ }^{11}$ J.J. Jia, T.A. Callcott, J. Yurkas, A.W. Ellis, F.J. Himpsel, M.G. Samant, J. Stöhr, D.L. Ederer, J.A. Carlisle, E.A. Hudson, L.J. Terminello, D.K. Shuh, and R.C.C. Perera, Rev. Sci. Instrum. 66, 1394 (1995).

${ }^{12}$ S. Okada and S. Saito, Phys. Rev. B 59, 1930 (1999).

${ }^{13}$ O. Gunnarsson, Rev. Mod. Phys. 69, 575 (1997).

${ }^{14}$ Y. Kopelevich, R.R. da Silva, J.H.S. Torres, A. Penicaud, and T. Kyotani, Phys. Rev. B 68, 092408 (2003).

${ }^{15}$ T.L. Makarova, K.-H. Han, P. Esquinazi, R.R. da Silva, Y. Kopelevich, I.B. Zakharova, and B. Sundqvist, Carbon 41, 1575 (2003).

${ }^{16}$ P.O. Lehtinen, A.S. Foster, A. Ayuela, A. Krasheninnkov, K. Nordlund, and R.M. Nominen, Phys. Rev. Lett. 91, 017202 (2003). 